\section{Impacto de la educación participativa en el índice de masa corporal y glicemia en individuos obesos con diabetes tipo 2}

\author{
Impact of participatory education on body mass \\ index and blood glucose in obese type- 2 diabetics
}

\author{
1 Unidad de Investigación \\ en Epidemiología Clínica, \\ Hospital de Especialidades, \\ Centro Médico Nacional \\ de Occidente, Instituto \\ Mexicano del Seguro Social, \\ Guadalajara,México. \\ 2 Centro de Estudios en \\ Salud, Población y Desarrollo \\ Humano, Departamento \\ de Ciencias Sociales, Centro \\ Universitario de Ciencias \\ de la Salud, Universidad \\ de Guadalajara, \\ Guadalajara,México. \\ Correspondencia \\ Carlos Enrique \\ Cabrera-Pivaral \\ San Juan Bosco 3782 \\ Col.Chapalita, Guadalajara \\ Jalisco, México. \\ carlose@megared.net.mx
}

\begin{abstract}
Exogenous obesity increases morbidity and mortality risk and has been associated with nutritional habits, which in turn can be affected by health education. Health education aims to promote patient participation in achieving behavior change and healthy lifestyles. The objective of this study is to show the advantages of participatory education in the modification of body mass index (BMI) in obese type-2 diabetics. A quasi-experimental study was performed with random allocation of two patient groups. The educational intervention was organized through a reflection-action process. BMI was measured at baseline and then monthly for 9 months during the intervention. The groups were analyzed by age and sex. Statistical analysis used the Student t test, with the mean difference for related groups. The control group showed a mean BMI of $33.89+1.96$ and a final BMI of $33.2 \pm 2.15$ ( $t: 22.4$; p: 0.16). The experimental group had an initial value of 33.63 \pm 2.12 and a final BMI of $31.54 \pm 1.71$; statistical difference: $(t: 11.55 ; p: 0.003)$. The participatory educational intervention thus helped improve the BMI in obese type-2 diabetics.
\end{abstract}

Health Education; Diabetes Mellitus; Body Mass Index
Carlos Enrique Cabrera-Pivaral 1,2

Guillermo González-Pérez 2

María Guadalupe Vega-López 2

Elva Dolores Arias-Merino 2

\section{Introducción}

La obesidad está acompañada de múltiples y graves consecuencias para la salud, además, se ha de reconocer que el riesgo de morbilidad y mortalidad 1,2 aumenta en la población obesa. La obesidad relacionada con factores nutricionales y estilos de vida se vincula con un exceso en los hábitos alimentarios, y estos con la educación para la salud. Esta última tiene como propósito la participación del diabético para promover comportamientos humanos y estilos de vida saludables, y del mismo modo propiciar cambios en el ambiente externo, dirigir la formación de recursos humanos y desarrollar investigación en este campo 3,4.

Como proceso, la educación para la salud se instrumenta a partir de la reflexión-acción; el educador y el educando efectúan un razonamiento acerca de los estilos de vida saludables. Todo ello mediante la entrevista y la acción organizada, partiendo del reconocimiento de sus ventajas y aciertos 4 .

El perfil del diabético tipo 2 se caracteriza por obesidad exógena y malos hábitos alimenticios que se relacionan con un ambiente externo que no participa en informar sobre su salud, ni motiva para adoptar y mantener hábitos y estilos de vida saludables.

La obesidad va a la par del aumento del riesgo de mortalidad en todas las edades; esta se incrementa hasta en un $40 \%$ en las enferme- 
dades coronarias. Los hábitos alimenticios inadecuados ocasionan trastornos en el perfil metabólico de los lípidos séricos. El 20\% de los pacientes diabéticos tiene alteraciones en el perfil de los lípidos 1 y esto es un factor de riesgo para los trastornos cardiovasculares 5 .

La aplicación de un plan educativo de tipo participativo, basado en la reflexión-acción sobre aspectos de la nutrición humana en el paciente diabético tipo 2, será el camino para el control del Índice de Masa Corporal (IMC).

\section{Material y métodos}

Se realizó un estudio cuasi experimental en 49 pacientes diabéticos obesos tipo 2, interesados en participar en un proyecto de intervención educativa durante un período de 9 meses. Todos ellos son usuarios de los servicios de salud de Medicina Familiar en el Instituto Mexicano del Seguro Social en Guadalajara, Jalisco, México durante 1999-2000. El propósito fue demostrar las ventajas de la educación participativa en el IMC de diabéticos tipo 2 con obesidad (IMC $>25$ y < 40) 6 .

Los pacientes a estudiar fueron convocados mediante una Asamblea en la Unidad de Medicina Familiar. De los asistentes aceptaron participar voluntariamente un $60 \%$, los cuales fueron asignados en forma aleatoria en dos grupos, uno para recibir la educación participativa (grupo experimento) y el otro, la educación tradicional (grupo control) 7.

Se tomó en cuenta la edad, el sexo, la escolaridad y los años de evolución de la diabetes 2 . Además, se considero el peso y talla con ropa ligera y sin zapatos utilizando una báscula y estadímetro de adulto calibrado diariamente. La obesidad fue reconocida con un IMC entre el rango de 25 a 406 .

La talla se determinó con aproximación al centímetro más cercano con el sujeto de pie. El IMC se calculó como el peso (en $\mathrm{Kg}$.) dividido entre la talla al cuadrado (en mm). Dichos datos no mostraron diferencias estadísticas entre ambos grupos antes de la intervención.

Se tomó una muestra de sangre venosa a todos los participantes, que descansaban en cama en una habitación. Se dejó reposar a temperatura ambiente por 30 minutos y, posteriormente, se centrifugó y midió la glucosa por duplicado en una unidad hospitalaria utilizando en método enzimático. El control de calidad se realizó según lineamientos de la Organización Mundial de la Salud, con curvas de calibración y variancia 1.

El grupo experimental se formó con 25 pacientes y el de control con 24 , quienes cubrieron un mínimo del 90\% de asistencia (32 sesiones).

\section{El programa educativo participativo}

El programa educativo participativo se desarrolló una vez por semana durante 9 meses, con una duración máxima de dos horas cada sesión, coordinado por una trabajadora social y un especialista en nutrición adiestrado en la metodología participativa. Los contenidos temáticos abordaron: conceptos y definiciones de la Diabetes Mellitus (2hrs.), anatomía (2hrs), fisiología (2hrs.), psicología y comportamiento humano (15 hrs.), complicaciones agudas y crónicas (10hrs.), nutrición básica y aplicada (10hrs.), grupos básicos de los alimentos y nutrimentos (10hrs.), dieta (6hrs.), autocontrol (10hrs.), ejercicio e higiene (6hrs.) y apoyo familiar (10hrs.). Las 82 sesiones temáticas giraron entorno a un momento reflexivo, con duración aproximada de 1.4 horas y a un momento de acción ( 0.6 hora) con desarrollo de talleres, trabajo en equipo y la discusión de las experiencias de aprendizaje con la guía de comportamiento dietético.

El proceso educativo participativo se caracterizó por: (a) el interés en los procesos y efectos; (b) la consideración del educando como un sujeto crítico y creativo; (c) la búsqueda del desarrollo de las habilidades y capacidades naturales del sujeto para transformar la realidad individual y social; (d) la comunicación a través del diálogo, el intercambio de ideas y la comparación de acciones entre los participantes; (e) la utilización de técnicas de educación y participación comunitarias y (f) la incorporación de la relación de los objetivos informativos y formativos 8 .

La reflexión consistió en un análisis crítico por parte de los participantes sobre las necesidades sentidas y expresadas con relación a su control metabólico, mediante técnicas de educación participativa que favorecieron la integración, comunicación y análisis general. La acción se caracterizó por el proceso de planeamiento y organización de actividades de autocuidado a través del equipo comunitario a fin de modificar los hábitos dietéticos y lograr el control metabólico. Los talleres en aula de aprendizaje favorecieron la construcción de una guía de comportamiento higiénico-dietético.

La guía de comportamiento surgió después de un taller con una dinámica educativa denominada lluvia de ideas, la cual permitió integrar un listado de hábitos a observar durante la vida cotidiana de los pacientes.

En general se desarrollaron cuatro niveles de abordaje: (1) El autodiagnóstico, que se constituyó mediante el conocimiento de la realidad en la cual el paciente diabético vive cotidiana- 
mente con su enfermedad y sus hábitos de alimentación. (2) La teorización, basada en los conceptos operativos de diabetes, nutrición y hábitos de alimentación. (3) La práctica, que consistió en talleres de aplicación de las leyes de la alimentación y la nutrición. (4) La autoevaluación realizada en dos etapas: la relacionada con el apego a la guía de cotejo de los hábitos de comportamiento dietético y la del control metabólico logrado durante el mes.

\section{La intervención educativa tradicional}

La intervención educativa tradicional en el grupo control se caracterizó por las estrategias comunicativas de tipo informativo con el apoyo de medios audio-visuales que permitían el intercambio de ideas. Se desarrolló una vez por semana durante 9 meses. Los contenidos temáticos fueron primero expuestos mediante conferencias magistrales y demostraciones con una duración aproximada de 1.2 horas cada una, para posteriormente analizar las experiencias con las guías de comportamiento dietético.

El propósito fue mantener el orden del proceso educativo, en el cual el docente informa al educando, reconociendo la necesidad de ciertas mejoras en el proceso y en donde los contenidos educativos son asignados con base en una jerarquización a priori efectuada por el docente.

Los temas y contenidos del curso fueron los mismos que se desarrollaron en el grupo de experimento. El aprendizaje en el grupo de metodología tradicional se inició con la recepción de conocimientos y memorización de los datos más importantes, como los nutrimentos, las calorías, el control metabólico, los hábitos higiénicos. La construcción y asignación de la lista de cotejo de los hábitos de comportamiento a seguir se elaboró con base en los contenidos y temas desarrollados por el profesor coordinador.

\section{Análisis estadístico}

Los resultados se expresan utilizando la media y desviación estándar, la prueba t para muestras relacionadas, además de la diferencia de medias para grupos relacionados.

El protocolo fue previamente autorizado por el Comité de Investigación y de Ética de la Institución y se obtuvo, asimismo, el consentimiento informado de los participantes, según el artículo 17 de la Ley General de Salud en materia de investigación en seres humanos.

\section{Resultados}

Datos generales de los grupos de estudio

El grupo experimental se conformó con 13 pacientes de sexo masculino (52.0\%) y 12 (48.0\%) del sexo femenino, y el grupo control con 11 de sexo masculino (45.8\%) y $13(54.2 \%)$ del sexo femenino $\left(\mathrm{x}^{2}=0.18 ; \mathrm{p}=0.67\right)$. La edad en el grupo experimento fue de $58.1 \pm 12.4$ años y en el grupo control fue de $57.8 \pm 8.7(\mathrm{t}=0.68 ; \mathrm{p}=$ $0.50)$. Con respecto a la escolaridad se observó que la moda en el grupo de experimento fue la primaria completa en el $44.0 \%$ de los casos y para el grupo de control fue la secundaria completa en el $41.7 \%$ de los pacientes $(t=2.79$; $\mathrm{p}=$ 0.09). Con relación a los años de evolución de la Diabetes Mellitus para el grupo experimental fue de $8.8 \pm 9.0$ años y para el grupo control de $11.3 \pm 11.8(\mathrm{t}=0.81 ; \mathrm{p}=0.57)$, sin mostrar diferencias en la medición inicial y final entre los grupos de estudio $(\mathrm{p}=0.57$ ) (Tabla 1$)$.

\section{Grupo de control}

El promedio del IMC es de $33.89 \pm 1.96$ con un rango de entre 30 a $36.5 \mathrm{Al}$ final de la intervención logra un promedio de $33.2 \pm 2.15$; con lo que demuestra una diferencia no significativa entre la medición inicial y final $(\mathrm{F}=22.4$; $\mathrm{p}=$ $0.16)$.

\section{Grupo de experimento}

En el grupo de experimento antes de la intervención educativa muestra un promedio de $33.63 \pm 2.12$ con un rango de 28 a 36 . Al final de la intervención educativa muestra un valor de IMC de $31.54 \pm 1.71$ lo que refleja una diferencia estadísticamente significativa $(\mathrm{F}=11.55$; $\mathrm{p}=0.003$ ) (Tabla 2).

\section{Comparación entre los grupos de estudio}

El IMC entre ambos grupos antes de la intervención demuestra una homogeneidad sin diferencia estadística ( $\mathrm{F}=0.19 ; \mathrm{p}=0.43$ ) (Tabla 2). Al efectuar un análisis estadístico entre el grupo de experimento y el de control después de la intervención educativa se observó una diferencia estadística significativa $(\mathrm{F}=9.27 ; \mathrm{p}=$ 0.0037).

En lo referente a los valores de glucosa en sangre en ayunas, según grupo de experimento y de control antes-después de la intervención, se observa una homogeneidad en los valores iniciales $(210.9 \mathrm{mg} / \mathrm{dl}$ con \pm 43.9 y 221.6 con \pm 83.2; $\mathrm{p}>0.05$ ), además de reconocer su distri- 
Tabla 1

Características generales de diabéticos II según grupo.

\begin{tabular}{|c|c|c|c|c|}
\hline \multirow[t]{3}{*}{ Variable } & \multicolumn{4}{|c|}{ Grupo } \\
\hline & \multicolumn{2}{|c|}{ Control } & \multicolumn{2}{|c|}{ Experimento } \\
\hline & $\mathrm{F}$ & $\%$ & $\mathrm{~F}$ & $\%$ \\
\hline Edad (en años) & 24 & 100,0 & 25 & 100,0 \\
\hline$>50$ & 5 & 20,8 & 7 & 28,0 \\
\hline $51-60$ & 10 & 41,7 & 7 & 28,0 \\
\hline+60 & 9 & 37,5 & 11 & 44,0 \\
\hline Género & 24 & 100,0 & 25 & 100,0 \\
\hline Femenino & 13 & 54,2 & 12 & 48,0 \\
\hline Masculino & 11 & 45,8 & 13 & 52,0 \\
\hline Años de evolución & 24 & 100,0 & 25 & 100,0 \\
\hline$>10$ & 15 & 62,5 & 16 & 64,0 \\
\hline $10-20$ & 4 & 16,7 & 7 & 28,0 \\
\hline+20 & 5 & 20,9 & 2 & 8,0 \\
\hline Escolaridad & 24 & 100,0 & 25 & 100,0 \\
\hline Primaria incompleta & 4 & 16,7 & 7 & 28,0 \\
\hline Primaria completa & 7 & 29,2 & 11 & 44,0 \\
\hline Secundaria & 10 & 41,7 & 5 & 20,0 \\
\hline Preparatoria + & 3 & 12,5 & 2 & 8,0 \\
\hline
\end{tabular}

Tabla 2

Índice de masa corporal en dos grupos antes y después de una intervención educativa participativa durante 9 meses de estudio. Guadalajara, Jalisco, México, 1999-2000.

\begin{tabular}{lcccc}
\hline $\begin{array}{l}\text { Índice de masa } \\
\text { corporal }\end{array}$ & \multicolumn{2}{c}{ Control } & \multicolumn{2}{c}{ Experimento } \\
& Pre & Post & & Post \\
\hline $28-29,9$ & 0 & 0 & 0 & 5 \\
$30-31,9$ & 4 & 7 & 7 & 10 \\
$32-33,9$ & 10 & 9 & 7 & 8 \\
$34-35,9$ & 5 & 5 & 7 & 2 \\
$36-37,9$ & 5 & 3 & 4 & 0 \\
Total & 24 & 24 & 25 & 25 \\
Promedio & 33,89 & 33,2 & 33,63 & 31,54 \\
D. estándar & 1,96 & 2,15 & 2,12 & 1,71 \\
Significancia & $f=22,4$ & $p=0,16$ & $f=11,55$ & $p=0,003$
\end{tabular}

Fuente: directa. bución normal mediante la representación de los valores z bajo la curva simétrica y mesocúrtica. Al finalizar la intervención se muestra una diferencia estadística a favor del grupo de experimento (147.6 con \pm 32.8 y $182.8 \pm 48.2$ ) (Tabla 3). Al efectuar una correlación entre el IMC y la glucosa se reconoce una influencia entre ambas; sugiriendo que los valores séricos y el IMC con el valor $p$ advertido indica que es poca la probabilidad de que la magnitud de la correlación ocurra por el azar $(r=0.72 ; p<0.05)$.

\section{Discusión}

La modificación del comportamiento del paciente diabético tipo 2 como resultado de la intervención comunicativo-educativa ${ }^{9}$, que condiciona sus hábitos y destrezas, tiene como objetivo mejorar el IMC, sin descartar el papel fundamental de otros factores sociales, este informe busca resaltar la importancia del proceso educativo para mejorar las condiciones de vida de los pacientes diabéticos 10 .

En la búsqueda por lograr el IMC normal al diabético, los trabajadores de la salud, incluido el enfermo, desarrollan un proceso de reflexiónacción en conjunto para estructurar un plan de autocuidado para el manejo del control adecuado de la glucosa 11,12. Valores, que si bien en el estudio no logran la normalidad, muestran disminuciones favorables para la salud, especialmente en el grupo que recibe la intervención participativa.

La disminución del IMC, la glucosa sanguínea y los lípidos séricos mediante una intervención educativo nutricional 13,14 en diabetes 2 con la modalidad tradicional de la educación 15 tiene inconvenientes y limitaciones, que en su mayoría se caracterizan por ser más informativos que formativos, donde se trata al educando como un objeto mediante una relación de tipo autoritaria, similar a la desarrollada en el grupo control y en la cual se reconocen algunos beneficios importantes en la postmedición. Sin embargo, hay que enfatizar que la educación participativa forma al paciente diabético, convirtiéndolo en un ser más reflexivo y comprometido para mejorar sus condiciones de salud y de vida.

El descenso prolongado y permanente de los niveles de glucosa y colesterol 14, aseguran la disminución de la severidad de la ateroesclerosis y del riesgo cardiovascular, objetivo alcanzado por el grupo de experimento en el caso del nivel de glucosa sanguínea, ya que logra disminuir $29.7 \%$ de su medición inicial. Sin embargo, y a pesar de que el proyecto no buscó 
como efecto principal prevenir lesiones clínicas, sus resultados deberán ser motivo de nuevas hipótesis para proyectos futuros, que desde la perspectiva de la educación y la cultura en salud logren vincular el beneficio clínico.

Las técnicas de motivación, participación y autocuidado 16 han demostrado que es más fácil desarrollar esquemas de tipo formativo con los pacientes diabéticos y que estos se traduzcan en el control de factores de riesgo 17,18. La intervención educativa favoreció el autocuidado y la conciencia social en salud, a través de fomentar el área de la educación para la salud y de la psicología social, al utilizar el co-monitoreo, el co-registro, la co-evaluación y el coreforzamiento con la finalidad de que el paciente se adhiera al tratamiento y mejore así sus condiciones de salud y de vida.

En los países en desarrollo, existe hoy en día acuerdo general sobre la importancia de los estilos de vida en la causalidad de los problemas de salud, así como también en la importancia de formar tempranamente estilos de vida saludables 11. Todos los países coinciden en poner en marcha programas de educación sanitaria con el fin de prevenir la enfermedad y las complicaciones mediante la modificación de los comportamientos humanos. El presente estudio ofrece una metodología educativa útil que da respuesta a cómo acercarse a los diabéticos a fin de que éstos participen en su autocuidado y modifiquen los valores de glucosa sanguínea y de la masa corporal. Así como un aporte relevante para enfrentar un problema de gran magnitud y trascendencia para la salud pública y los servicios de salud en América Latina.

Compartimos la afirmación de Ulrich 19, en cuanto a que el objetivo de la educación sanitaria es estimular y desarrollar al paciente diabético tipo 2, sin descuidar lo que Freire 20 menciona: al hombre como esencialmente crítico para la transformación de la naturaleza en un beneficio razonado y creativo.

La influencia e impacto de las intervenciones educativas en salud, a través de vincular la teoría con la práctica 21 , definen las estrategias de intervención, enfatizando que los proyectos educativos en grupo generan más ventajas en el aprendizaje 9,22, como lo reportado en este estudio que valora el proceso grupal sobre el proceso individual de tipo informativo enciclopedista.

Leino 5 y Logue 23 analizan cómo el nivel de escolaridad entre los diabéticos tipo 2, define sus estilos de vida, además de caracterizar el control metabólico de su enfermedad. En nuestro estudio la escolaridad no mostró diferencia en los grupos de estudio, pero seguramente
Tabla 3

Glucosa en sangre de ayunas en dos grupos antes y después de una intervención educativa participativa durante 9 meses. Guadalajara, Jalisco, México, 1999-2000.

\begin{tabular}{lrrrr}
\hline \multirow{2}{*}{ Glucosa (mg/dl) } & \multicolumn{2}{c}{ Control } & \multicolumn{2}{c}{ Experimento } \\
& Pre & Post & Pre & Post \\
\hline$>0$ & 0 & 0 & 0 & 0 \\
$80-139$ & 0 & 3 & 0 & 10 \\
$140-199$ & 13 & 13 & 10 & 14 \\
$200-259$ & 5 & 5 & 11 & 1 \\
$260-319$ & 4 & 3 & 4 & 0 \\
$320-379$ & 1 & 0 & 0 & 0 \\
$380-439$ & 0 & 0 & 0 & 0 \\
+440 & 1 & 0 & 0 & 0 \\
Total & 24 & 24 & 25 & 25 \\
Promedio & 221,66 & 182,87 & 210,92 & 147,6 \\
D. estándar & 83,18 & 48,22 & 43,99 & 32,82 \\
Significancia & $f=2,51$ & $p=0,06$ & $f=7,53$ & $p=0,0005$ \\
\hline
\end{tabular}

Fuente: directa.

condiciona la información y la formación de hábitos en salud; factores básicos para planear programas de promoción de la salud 4,12,13,21.

El beneficio de recibir una orientación educativa temprana, adecuada y oportuna para el cuidado y autocontrol de la dolencia 24 ha sido un acuerdo aceptado por instituciones de servicios de salud; producto de reconocer que las personas sanas cuyo conocimiento de la nutrición y diabetes, incluso quienes la padecen, suele ser precario, frecuentemente sesgado y pleno de tabúes, errores y distorsiones $22,23,25$. Esto limita la capacidad del diabético para tomar decisiones sobre la importancia de su participación activa en el control de su IMC y, por lo tanto, en la asistencia a programas educativos.

El tamaño muestral y la selección de los participantes por su propio interés no permite establecer inferencias y puede generar un sesgo, el cual ha sido controlado mediante el grupo de referencia, sin embargo, se logran observar resultados valiosos para grupos con características similares; motivo por el cual se continuará con proyectos que permitan sustentar la inferencia estadística en poblaciones con características similares.

Finalmente, la intervención educativa participativa, mediante la reflexión-acción del paciente diabético tipo 2, ofrece beneficios en el control de la glucosa sanguínea y posteriormente en el IMC. 


\section{Resumen}

La obesidad eleva el riesgo de morbilidad y mortalidad y se ha relacionado con los hábitos alimentarios y estos con la educación en salud. Esta última tiene como propósito promover la participación de los enfermos en mejorar los comportamientos humanos y estilos de vida saludables y mostrar las ventajas de la educación participativa en la modificación del Índice de Masa Corporal (IMC) en el diabético obeso tipo 2. Se realizó un estudio cuasi experimental con asignación aleatoria de dos grupos de pacientes diabéticos obesos. La intervención educativa-participativa se organizó mediante el proceso de reflexión-acción. Se efectuaron mediciones del IMC basal y mensuales durante los 9 meses de la intervención educativa. Los grupos fueron controlados tomando en cuenta edad y sexo. El análisis estadístico se efectuó con el estadígrafo de t de students con diferencia de media para grupos relacionados. El grupo control logra un valor promedio de IMC basal de $33.89 \pm 1.96$ y al final de $33.2 \pm 2.15$ ( $t: 22.4$; $p$ : $0.16)$, el grupo de experimento con valor inicial de $33.63 \pm 2.12$ y final de $31.54 \pm 1.71$ ( $t: 11.55 ; p=0.003)$. La intervención educativa participativa contribuye a mejorar el nivel de IMC en los diabéticos obesos 2.

Educación en Salud; Diabetes Mellitus tipo 2; Índice de Masa Corporal

\section{Colaboradores}

C. E. Cabrera-Pivaral generó la idea principal del estudio y su diseño de intervención. Cada uno de los coautores participaron en el desarrollo; G. GonzálezPérez se responsabilizó de la recolección de datos, base de datos y su análisis estadístico. La discusión fue propuesta y desarrollada por M. G. Vega-López y E. D. Arias-Merino. El primer borrador del manuscrito fue por el autor principal y la versión final por el resto de coautores.

\section{Referencias}

1. Secretaría de Salud, México. Encuesta nacional de enfermedades crónicas. México DF: Dirección General de Epidemiología; 1995.

2. Shepherd J, Weissberg P. Time to treat: beyond survival, preventing recurrence of acute coronary syndromes. Atherosclerosis 1999; 147 Suppl:3-10.

3. Novoa MA. Currículum y plan de estudios de la Maestría en Educación para la Salud. Guadalajara: Universidad de Guadalajara; 1984.

4. Salleras SML. Educación sanitaria. Madrid: Díaz de Santos; 1990.

5. Leino M, Raitakari OT, Porkka KV, Taimela S, Viikar JS. Associations of education with cardiovascular risk factors in young adults. Int J Epidemiol 1999; 28:667-75.

6. James WPT, Ferro-Luzzi A, Waterlow JC. Definition of chronic energy deficiency in adults. Report of a working party of the International Dietary Energy Consultative Group. Eur J Clin Nutr 1988; 42:969-81.

7. Campbell D, Stanley J. Diseños experimentales y cuasi experimentales en la investigación social. Buenos Aires: Amorrortu; 1993.

8. Kaplun M. El comunicador Popular. Buenos Aires: Humanitas; 1990.

9. Schwandt P, Geiss HC, Ritter MM, Ublacker C, Parhofer KG, Otto C. The prevention education program. A prospective study of the efficacy of family oriented life style modification in the reduction of cardiovascular risk and disease: design and baseline data. J Clin Epidemiol 1999; 52:791800.

10. Racette SB, Weiss EP, Obert KA, Kohrt WM, Holloszy JO. Modest lifestyle and glucose tolerance in obese African American. Obes Res 2001; 9:348-55.

11. Lai SW, Li TC, Ng KC. Body mass index and its related factors in the elderly. Ann Acad Med Singapore $2001 ; 30: 397-400$

12. Keller U. Health Education and nutrition. Schweitz Med Wochenschr 1991; 121:1014.

13. Kustner E, Dancygier H. Efficacy an efficiency of ambulatory diabetes patient education. Dtsch Med Wochenschr 2000; 125:1280-1.

14. Bemelmans WJ, Broer J, De Vries JH, Hulshof KF, May JF, Meyboom JB. Impact of Mediterranean diet education versus posted leaflet on dietary habits and serum cholesterol in a risk population cardiovascular disease. Public Health Nutr 2000; 3:273-83.

15. Herd A. Relation of clinical benefit to metabolic effects in lipid-lowering therapy. Am J Cardiol 1998; 82 (6 Suppl 1):22M-5M.

16. Hershowitz R. Education and behavior in diabetes care. Diabetic Med 1990; 7:633.

17. Ko GT, Wu MM, Tang WHP, Chan $\mathrm{CH}$, Chen R. Body mass index profile in Hong Kong Chinesse adults. Ann Acad Med Singapore 2001; 30:393-6.

18. Woo J, Ho SC, Sham A. Longitudinal changes in body mass index and body composition over 3 years and relationship to health outcomes in Hong Kong Chinese age 70 and older. J Am Geriatr Soc 2001; 49:737-46.

19. Ulrich A. The integration of health education to patient care. New York: ADA; 1984. 
20. Freire P. Extensión o comunicación. La concientización en el medio rural. México DF: Siglo XXI; 1981.

21. Steckler A, Allegrante JP, Altman D. Health education intervention strategies. Health Educ Q 1995; 22:307-28.

22. Turnin MC, Bourgeois O, Cathelineau G, Leguerrier AM, Halami S, Banon D. Multicenter randomized evaluation of a nutritional education software in obese patients. Diabetes Metab 2001; 27:139-47.

23. Logue EE, Smucker WD. Obesity management in primary care: changing the status quo. J Fam Pract 2001; 50:520.

24. Van der Sande MA, Walraven GE, Milligan PJ, Banya WA, Ceesay SM. Family history: an opportunity for early intervention. Bull World Health Organ 2001; 79:321-8.

25. Potter MB, Vu JD, Croughan MM. Weight management want from their primary care physicians. J Fam Pract 2001; 50:513-8.

Recibido el 26/Feb/2002

Versión final presentada el 27/Ago/2003

Aprobado el 24/Oct/2003 